\title{
La Percepción de las Mujeres hacia las Acciones de Prevención, Denuncia y Erradicación del Hostigamiento Sexual en una Escuela del Instituto Politécnico Nacional en México
}

\author{
Claudia A. Hernández ${ }^{(1)}$, Martha Jiménez ${ }^{(1)}$ y Silvia Galicia ${ }^{(2)}$ \\ (1) Instituto Politécnico Nacional, Unidad Profesional Interdisciplinaria de Ingeniería y Ciencias Sociales y \\ Administrativas (UPIICSA), Sección de Estudios de Posgrado e Investigación. Av. Té 950, Iztacalco, \\ Granjas, 08400, México, D.F. - México (e-mail: cahernandezh@ipn.mx, majimenez@ipn.mx) \\ (2) Instituto Politécnico Nacional, Escuela Superior de Comercio y Administración unidad Tepepan, \\ Av. Manuel Carpio 471, Miguel Hidalgo, Plutarco Elías Calles, 11340, México, DF. - México \\ (e-mail: sgaliciav@ipn.mx).
}

Recibido Abr. 1, 2015; Aceptado May. 22, 2015; Versión final Jun. 2, 2015, Publicado Dic. 2015

\begin{abstract}
Resumen
El objetivo del trabajo es analizar la percepción que tienen las estudiantes del Instituto Politécnico Nacional en México de las acciones implementadas que permiten prevenir, sancionar y erradicar el hostigamiento sexual, y para diagnosticar la adecuada o nula presencia y efectividad de las mismas en la unidad académica. La metodología que se empleó está basada en un estudio de caso de tipo descriptivo y transversal. La recolección de los datos se realizó por medio de un cuestionario que se aplicó a una muestra de 901 mujeres estudiantes en una escuela del Instituto Politécnico Nacional en México. Los resultados muestran que el desconocimiento de los procedimientos para denunciar, la falta de protocolos de intervención y la escasa capacitación de los cuerpos directivos, generan situaciones que vulneran la vida y derechos de las estudiantes.
\end{abstract}

Palabras clave: hostigamiento sexual; violencia de género; mujeres estudiantes; denuncia de crímenes

\section{Perception of Women toward the Actions of Prevention, Report and Eradication of Sexual Harassment in a School of the National Polytechnic Institute in México}

\begin{abstract}
The objective of this work is to analyze the perception that women students of a school of the National Polytechnic Institute in Mexico, of the actions implemented to prevent, punish and eradicate sexual harassment, to diagnose the proper or null presence and effectiveness of those actions in the academic unit. The methodology that was used is based on a case study of descriptive and transversal type. Data collection was gathered by means of a questionnaire that was applied to a sample of 901 women students of a school of the National Polytechnic Institute. The results show that ignorance of the procedures for reporting harassment, the lack of intervention protocols and the lack of training of the governing bodies, generate situations that violate student's life and rights.
\end{abstract}

Keywords: sexual harassment; gender-based violence; female students; crime reporting 


\section{INTRODUCCIÓN}

México se ha comprometido con los objetivos de las convenciones, cumbres y tratados internacionales, derivado de lo anterior en el año 2001 se promulga la Ley del Instituto Nacional de las Mujeres y se crea el Instituto Nacional de las Mujeres (INMUJERES), institución a nivel federal que busca establecer una cultura de igualdad libre de violencia y discriminación apoyando el desarrollo integral de las mujeres, además promueve el ejercicio pleno de los derechos tanto para hombres como para mujeres. En el 2011 la Encuesta Nacional sobre la Dinámica de las Relaciones en los Hogares, les preguntó a una muestra amplia de mujeres mayores de 15 años, si durante su vida escolar habían sido agredidas por algún compañero, maestro o autoridad del plantel educativo. La encuesta encontró que el $42 \%$ de las encuestadas habían sufrido violencia sexual, proposiciones de relaciones sexuales a cambio de calificaciones, caricias 0 manoseos sin consentimiento o represalias por no acceder a las propuestas o en el último de los casos las habían forzado a tener relaciones sexuales.

La presencia desde el 2001 del INMUJERES en México ha permitido que las mujeres vayan tomando presencia y sean tomadas en cuenta desde la planeación a nivel federal tanto en el tema de presupuestos como en el desarrollo de intervenciones que permitan un trato equitativo entre mujeres y hombres. Actualmente, en México se han promulgado leyes que permiten entender y sancionar el tema de la violencia, como son la Ley General de Acceso de las Mujeres a una vida Libre de Violencia (2014) que promueve un Programa Integral y un Sistema Nacional de Prevención, Atención, Sanción y Erradicación de la Violencia contra las mujeres. A su vez, en México actualmente se encuentra en funcionamiento el Programa Nacional para la Igualdad de Oportunidades y no Discriminación contra las Mujeres 2013-2018.

El Instituto Politécnico Nacional (IPN) es un organismo desconcentrado de la Secretaría de Educación Pública, en el año de 2008 establece el acuerdo del Programa Institucional de Gestión con Perspectiva de Género con el objetivo central de promover y fomentar las condiciones que posibiliten la no discriminación y la igualdad de oportunidades entre hombres y mujeres. En mayo de 2012 este programa se convirtió en la Unidad Politécnica de Gestión con Perspectiva de Género, y ha trabajado de forma intensa, implementando la campaña de Prevención, Atención y Sanción para Erradicar el Acoso y el Hostigamiento en los Ámbitos Laboral y Escolar, se cuenta con un sistema de denuncia segura por medio de un portal web, de tal manera que tanto estudiantes como trabajadores pueden manifestar si están siendo víctima de algún tipo de violencia de género o de discriminación (Rodríguez, 2013).

La implementación de las acciones que permiten prevenir, denunciar y erradicar el hostigamiento sexual (HS) es una obligación que tienen las instituciones de proteger y brindar espacios saludables de convivencia y libres de violencia para sus estudiantes. Sin embargo no se tiene evidencia de la evaluación, seguimiento y satisfacción de las mismas en la comunidad estudiantil, además de identificar su presencia o carencia. El estudiar la percepción de las estudiantes tiene su origen en el número de denuncias presentadas hasta el año 2013 en la Oficina del Abogado General del IPN, siendo la escuela en donde se realizó el estudio una de las tres con más denuncias, la información fue proporcionada por el Instituto Federal de Acceso a la Información y Protección de Datos (IFAI). Lo anterior, aunado al escaso conocimiento que se tiene del tema en las unidades académicas en donde se percibe el escaso entrenamiento, protocolos con poca precisión y una sensación de una posible falta de atención a los posibles casos de violencia sexual.

En México existen pocas investigaciones que consideren el tema de hostigamiento sexual en estudiantes y la evaluación de sus mecanismos de denuncia, en la búsqueda de información se encontró que Rosas y López (2011) indagan sobre el hostigamiento y acoso en el ámbito laboral y escolar, sin embargo, el estudio no permite identificar en específico la situación de cada escuela, además de que levanta datos tanto de alumnas como de trabajadoras. Por otro lado, Ochoa (2008) encuentra en sus investigaciones que la debilidad de los programas de Tutoría es el género, en un estudio empírico encontró que en algunas ocasiones los tutores tienden a sexualizar su interacción con las alumnas, esto provoca que las jóvenes dejen de acudir a la asesoría, arriesgan su calificación y desertan de su clase. Por otro lado, las autoras Buquet, Cooper, Mingo y Moreno (2013) advierten la emergencia de retomar el estudio a profundidad del fenómeno del hostigamiento sexual, analizan las tres poblaciones que conforman la Universidad Nacional Autónoma de México (UNAM): la estudiantil, la académica y la administrativa, encuentran zonas críticas de inequidad, en donde se descubren prácticas machistas que ponen en desventaja a las mujeres de la comunidad universitaria; sin embargo también se hallaron casos de hombres que son víctimas de hostigamiento sexual. Es importante señalar que una cultura del silencio prohíbe y censura el dar la apertura necesaria para discutir los asuntos relacionados con el hostigamiento sexual, éste tema puede estar acompañado de los silencios y de la vergüenza que puede ocasionar (Oliver, 2011; Dhlomo, Mugwen, Shoniwa, Maunganidze y Sodi, 2012).

Estudiar el hostigamiento sexual y la percepción que tienen las estudiantes sobre las medidas de intervención y mecanismos de denuncia es importante para detectar la eficacia de las estrategias de 
intervención implementadas en la institución y de esta manera evaluar las mismas para así reconocer el avance o retroceso, lo anterior para proponer estrategias de mejora. Bajo el anterior contexto, el objetivo del trabajo es analizar la percepción que tienen las estudiantes de una escuela de educación superior en México sobre las acciones implementadas que permiten prevenir, sancionar y erradicar el hostigamiento sexual, para diagnosticar su adecuada o nula presencia y efectividad de las mismas en la unidad académica.

\section{EL HOSTIGAMIENTO SEXUAL Y LAS FORMAS DE PREVENCIÓN}

El hostigamiento sexual contempla todos aquellos avances sexuales no deseados, así como aquellos comentarios sexuales que pueden estar acompañados de gestos que se pueden presentar en cualquier entorno (Mitchell, Ybarra y Korchmaros, 2014; Fasting, Chroni y Knorre, 2014). En este sentido, el hostigamiento sexual es cualquier forma de conducta no deseada de naturaleza sexual, las expresiones pueden ser físicas, verbales y no verbales, siendo las físicas consideradas como las más graves, incluyen contacto físico, avances sexuales no deseados, asalto sexual y puede llegar a convertirse en violación. El verbal se expresa mediante las peticiones de favores sexuales, comentarios sobre las partes del cuerpo, chistes e insinuaciones de tipo sexual y el no verbal es la exhibición de imágenes con contenido pornográfico (Jackman, 2006; Maguire, 2010; Hill y Kearl, 2011).

El hostigamiento sexual es una situación de quid pro quo (una cosa por otra) en donde un profesor desarrolla ambientes hostiles, sometiendo a los alumnos a conductas sexuales no deseadas, creando un ambiente amenazador, hostil u ofensivo (Krauss, Krauss, O`Day y Rente, 2005; Gelfand, Fitzgerald y Drasgow, 1995; Paludi, et al., 2006).). Los comportamientos tienen la intención de degradar al género, puede incluir la visualización de fotos con cuerpos desnudos, hacer chistes con contenido sexual y efectuar comentarios obscenos sobre el cuerpo de alguien, palmadas, pellizcos, rozar el cuerpo de otro, objetos sexualmente sugestivos como libros, revistas, carteles, dibujos animados y correos electrónicos (Sbraga y O'donohue, 2000; Kalof, et al, 2001; Pina, Gannon y Saunders, 2009; Vázquez, Torres y Otero, 2012). Algunas de las conductas de acoso de género son los comentarios insultantes, hostiles y degradantes que se hacen hacia las mujeres, tocar o acariciar, realizar insinuaciones sexuales y propuestas para tener relaciones sexuales y por último la coerción sexual acompañada con el soborno y el chantaje sexual (Angelone, Mitchell y Carola, 2009; Mitchell, Ybarra y Korchmaros, 2014; Fasting, Chroni y Knorre, 2014).

Algunos estudios han encontrado que en su mayoría las mujeres son más propensas a ser víctimas del hostigamiento sexual que los hombres (Reilly, Lott y Gallogly, 1986; Sbraga y O'donohue, 2000; Yik, Cheung, Choi y Au, 1996; Jackman, 2006; Rahimi y Liston, 2011; Tang, Bursik y Gefter, 2011; Abuya, Onsomu, Moore y Sawge, 2012). El machismo hegemónico combina las relaciones en diferentes formas, el producto histórico de la reproducción cultural en la sociedad se exhibe en actos de servidumbre y sujeción. Sus expresiones se demuestran por medio de diversas formas de violencia (Castañeda y Castaaneda, 2002).

La comunicación es un factor por el cual se negocia el intercambio de favores sexuales, de dinero, de seguridad en la escuela o en el empleo a cambio de aceptar el hostigamiento sexual (Jones y Remland, 1992), el hostigamiento sexual sigue teniendo un impacto perjudicial sobre las experiencias educativas de muchos estudiantes universitarios, éstas provocan que los alumnos se sientan molestos, incómodos, enojados y decepcionados de su estancia en la Universidad (Hill y Silva, 2005), estudios demuestran que las mujeres y las personas con formación previa en materia de hostigamiento sexual rechazan más los mitos como el sexismo, la hostilidad y las actitudes tradicionales hacia las mujeres (Lonsway, Cortina y Magley, 2008).

Oliver (2011) señala que la universidad sigue siendo del domino predominantemente masculino, en las que se producen distintas formas de misoginia y de discriminación, que afecta a las estudiantes y a las mujeres académicas, a su vez, las diferentes formas de violencia se convierten en barreras que tienen impacto negativo. Existen expresiones que son empleadas como un lenguaje misógino que les recuerda a las mujeres lo complejo que significa la vida escolar y si a lo anterior se le agrega cuestiones de raza, origen étnico, preferencia sexual o religión, todo se mezcla y se convierte para algunas mujeres un terrorismo sexual (Rahimi y Liston, 2011).

Los programas de prevención son necesarios para identificar las oportunidades en donde se puedan desarrollar estrategias adecuadas, para ello se necesita contar con evidencia sustraída de información de evaluaciones realizadas al fenómeno de la violencia sexual, lo anterior para desarrollar acciones orientadas a prevenir y abatir la violencia sexual en la escuela y se esta forma proteger a los jóvenes y cuidarlos de ambientes hostiles que atenten contra su integridad. También, se requiere la existencia de un clima de respeto y confianza, en el cual los estudiantes, se sientan libres y seguros de que la escuela los apoyará en caso de que perciban algún acto que lastime su sexualidad. Sin embargo, toda acción emprendida requiere 
ser evaluada en un período de tiempo determinado (DeGue, 2014). El hostigamiento sexual que se presentan en las universidades refleja que todavía hay mucho trabajo por hacer para fomentar ambientes escolares libres de prejuicios y de violencia de género, de ésta manera todos los alumnos podrán sobresalir en espacios mucho más saludables y libres de violencia (Hill y Kearl, 2011).

Otro elemento que funciona como preventivo es la promoción de climas de empoderamiento en donde los estudiantes reciben información y son capaces de diferenciar las diversas situaciones que generan hostigamiento sexual (Bond, 1995). Además, para que las políticas funcionen y tengan credibilidad es necesario que los estudiantes tengan confianza en los medios de información, contar con un asesoramiento y apoyo informal para las víctimas que han sufrido hostigamiento sexual eviten pasar por un proceso largo y burocrático, mientras que las políticas no se utilicen corren el riesgo de socavar su credibilidad y puede pensarse que las mismas sólo están diseñadas para funcionar como escaparates que usan las escuelas para dar a conocer a la sociedad que se está haciendo frente al hostigamiento sexual (Thomas, 2004).

La formación anti hostigamiento sexual es un mecanismo que ayuda en la prevención (Fusilier y Penrod, 2014), por otro lado, los programas de educación preventiva deben de incluir: 1) definiciones del hostigamiento sexual y violencia sexual, 2) explicación de la política de la escuela, procedimiento y protocolos en caso de ser víctima, 3) direcciones de contacto que ofrecen apoyo y 4) acciones afirmativas que motiven a los estudiantes a denunciar actos de violencia (Osborne, 2013).

En el presente trabajo se define al hostigamiento sexual como las conductas que manifiestan o expresan acciones con contenido sexual, las cuales provocan incomodidad y enojo en los estudiantes. Además de ser una demostración de poder que puede ejercer una persona sobre otra, lo cual puede ocasionar angustia, miedo, estrés e impotencia, generalmente las expresiones vienen acompañadas de un intercambio sexual a cambio de un favor académico o económico. Las acciones afirmativas que se requieren para prevenirlo, denunciarlo y erradicarlo son diversas, desde las campañas hasta los procesos de denuncia segura, pero la eficacia de las políticas y estrategias las perciben los estudiantes y por lo tanto se les debe de dar un seguimiento y evaluación de las mismas

\section{MATERIALES Y MÉTODOS}

Es un estudio transversal y descriptivo, se utilizó como instrumento una encuesta con ocho preguntas en materia de hostigamiento sexual, con escala tipo Likert en donde 1 = totalmente en desacuerdo y $5=$ totalmente de acuerdo, a una muestra de 901 estudiantes exclusivamente mujeres de una población total de trece mil alumnos, de los cuales el $60 \%$ son hombres. El instrumento obtuvo alfa de Cronbach de 0.860 dicho coeficiente muestra una buen nivel de confiablidad. El muestreo que se utilizó fue aleatorio, la aplicación fue en el mes de octubre de 2013, se utilizó la estrategia de estudio de caso (Yin, 2003). Las preguntas se redactaron considerando a los autores Rahimi y Liston (2011), DeGue (2014), Hill y Kearl (2011), Bond (1995), Thomas (2004) y Osborne (2013).

1.- ¿En la escuela existe un procedimiento lo suficientemente claro para denunciar el hostigamiento sexual (HS)?

2.- ¿En la escuela existen redes de apoyo contra la violencia de género y que apoyan los casos de HS?

3.- ¿Conozco las vías de denuncia en caso de ser víctima de HS?

4.- ¿En la escuela se hace énfasis en promover las campañas en contra de la violencia contra las mujeres y HS?

5.- ¿Los profesores en sus clases fomentan el aprendizaje con relación al tema de HS?

6.- ¿Conozco las sanciones que se aplican en los casos de hostigamiento sexual?

7.- ¿Las campañas han ayudado para no ser víctima?

8.- ¿En la escuela existe alguna autoridad que inspira confianza para contarle situaciones de hostigamiento sexual?

Durante la aplicación del instrumento se hizo la observación de que la información proporcionada sería usada de manera confidencial y la estudiante estaba en su derecho de negarse a responder el instrumento. Las participantes que accedieron no se les cuestionó su tenían algún conocimiento previo sobre las campañas que previenen el hostigamiento sexual y el significado de éste.

\section{LAS PARTICIPANTES}

Las participantes en el estudio debían de tener las siguientes características: a) alumnas inscritas en a partir del segundo semestre. Se entrenó a un grupo de seis encuestadores del tal forma que se aplicó el instrumento en ambos turnos. Los datos fueron analizados con el software SPSS versión 20 . El cálculo de la muestra se realizó teniendo en cuenta el tamaño de la población $\mathrm{N}=4,314$ mujeres la muestra se calculó considerando un intervalo de confianza de 5 y un nivel de confiabilidad del $95 \%$, con un $p=50 \%$ y $q=1-p=$ $50 \%$, obteniéndose como resultado 384, la muestra generadora de datos fue de 901 . 


\section{RESULTADOS}

La edad de las estudiantes se encuentra en una media de 21 años con una desviación estándar de 2.24 años. El $70 \%$ de las mujeres están inscritas en el turno matutino y 30\% de las participantes en el vespertino. La carrera que más participó en el estudio fue la Licenciatura en Administración Industrial, con el 68.5\%; seguida de Ingeniería Industrial con 15\%; Ingeniería en Informática con el 9\%; la Licenciatura de Ciencias de la Informática con el 5\%; y sólo 3\% de Ingeniería en Transporte.

A las alumnas se les preguntó si conocían algún procedimiento lo suficientemente claro que les permitiera denunciar casos de hostigamiento sexual, $47 \%$ de las participantes dijeron no estar de acuerdo, 32\% dijo estar ni de acuerdo ni en desacuerdo, lo que hace dudar de que conozcan el proceso a seguir, sólo $21 \%$ de las alumnas señalaron que si conocen el proceso para denunciar. La existencia de redes de apoyo para combatir, prevenir y erradicar la violencia son fundamentales para que la escuela no vaya sola en el complejo camino para instaurar mecanismos de prevención y su momento de sanción ante los actos que en ocasiones son hostiles, a las alumnas de la escuela se les interrogó si han percibido la presencia de las redes de apoyo, 37\% dijo rotundamente que no sabía, mientras que un 38\% no estaba ni de acuerdo ni en desacuerdo y un $23 \%$ dijo que sí tenía el conocimiento de que estuvieran trabajando las redes de apoyo.

Tener claras las vías de denuncia es un mecanismo que apoya para que el hostigador sexual, tenga el conocimiento de que si continúa con esa conducta, la institución respaldará a las víctimas y las apoyará durante todo el tiempo que dure el proceso de la denuncia. A las alumnas que accedieron a responder el cuestionario se les consultó si sabían de la operación y existencia de las vías de denuncia, se encontró que $63 \%$ de las mujeres desconocen el método de denuncia.

El promover campañas que ayuden a prevenir, sancionar y erradicar la violencia contra las mujeres, es un cuestionamiento que se le hizo a las estudiantes, con la finalidad de reconocer la magnitud del espectro de acción de cada uno de los programas de sensibilización, al respecto, $40 \%$ de las participantes dicen desconocer las campañas. Además, se indagó si las alumnas tenían conocimiento sobre las sanciones que se aplican en la escuela en los casos de hostigamiento sexual, se halló que el $63 \%$ de las alumnas dice desconocer las posibles sanciones, hecho que invita a la reflexión, 25\% no tiene una orientación clara hacia su respuesta y sólo $10 \%$ dijo que sí sabía de los posibles castigos que se le puede imponer a un acosador u hostigador. Los profesores son buenos emisarios para comunicar, sensibilizar y fomentar actividades que ayuden a los jóvenes a reconocer el tema de hostigamiento sexual, así como los métodos de denuncia y medidas de prevención, al respecto, la investigación arroja como resultado que 55\% de las alumnas no está de acuerdo, sólo $26 \%$ de ellas reconoce el esfuerzo de los académicos por apoyar las acciones orientadas a promover la denuncia.

A las alumnas que respondieron el instrumento se les preguntó si ellas consideraban que las campañas que promueve la escuela las han apoyado para no ser víctimas del hostigamiento sexual, 38\% de las jóvenes señalaron que las campañas no ayudan en mucho, $22 \%$ mencionó que sí y el resto se mostró indiferente a su respuesta. A su vez, se indagó si las estudiantes perciben si existe alguna autoridad que les inspire confianza para poder contarle y denunciar algún tipo de acto o hecho que las haya hecho sentir mal, 39\% respondió que no estar de acuerdo, el 31\% ni de acuerdo ni en desacuerdo y el 30\% dijo que sí. (Ver figura 1).

\section{DISCUSIÓN}

Considerando los resultados es necesario mencionar que se requiere de una campaña con más amplio espectro que procure la transferencia de conocimientos respecto al tema, la concientización que se adquiere de los procesos de aprendizaje de los cursos o talleres de ayuda, son para conocer la parte conceptual relacionada con los tipos de violencia a los que se puede enfrentar una joven durante su tránsito en la escuela. Por lo que se sugiere que en las universidades se tengan grupos de mujeres debidamente entrenadas para enfrentar los problemas que se originan cuando se presentan situaciones de hostigamiento sexual, lo cual es equivalente a contar con escudos protectores que ayudarán a fomentar la denuncia bajo el amparo de las políticas institucionales.

De acuerdo con Rodríguez (2013) se deben de fomentar en las asignaturas el aprendizaje del tema del hostigamiento sexual y sus formas de presentarse en la escuela, además es imprescindible que se capacite y sensibilice a los académicos en temas de equidad de género, derechos humanos, cultura de la legalidad, ética, y resolución de conflictos, asimismo que las autoridades fomenten la creación de espacios saludables y de convivencia integral. Es una realidad que la escuela tiene la responsabilidad de proteger a los estudiantes contra el hostigamiento sexual. Pues la universidad, en este caso el IPN debe de proporcionar un ambiente pacífico, y debe de informar a las estudiantes sobre lo que deben de hacer en caso de ser víctimas de hostigamiento sexual, para de esta forma disminuir el fenómeno del hostigamiento y hostigamiento sexual (Maguire, 2010). 


\section{- En desacuerdo/Totalmente en desacuerdo $\square$ Ni de acuerdo ni en desacuerdo \\ - Totalmente de acuerdo/De acuerdo}

1. Existencia de un procedimiento claro que permite denunciar

2. Redes de apoyo en contra del acoso sexual

3. Conoce las vías de denuncia

4. En la escuela se hace énfasis en promover las campañas contra la violencia contra las mujeres

5. Los profesores fomentan el aprendizaje de lo qué es el acoso y cómo denunciarlo

6. Conoce las sanciones que se aplican en caso de acoso sexual

7. Las campañas ayudan para no ser víctima

8. En la escuela existe alguna autoridad que inspira confianza para contarle situaciones de acoso sexual

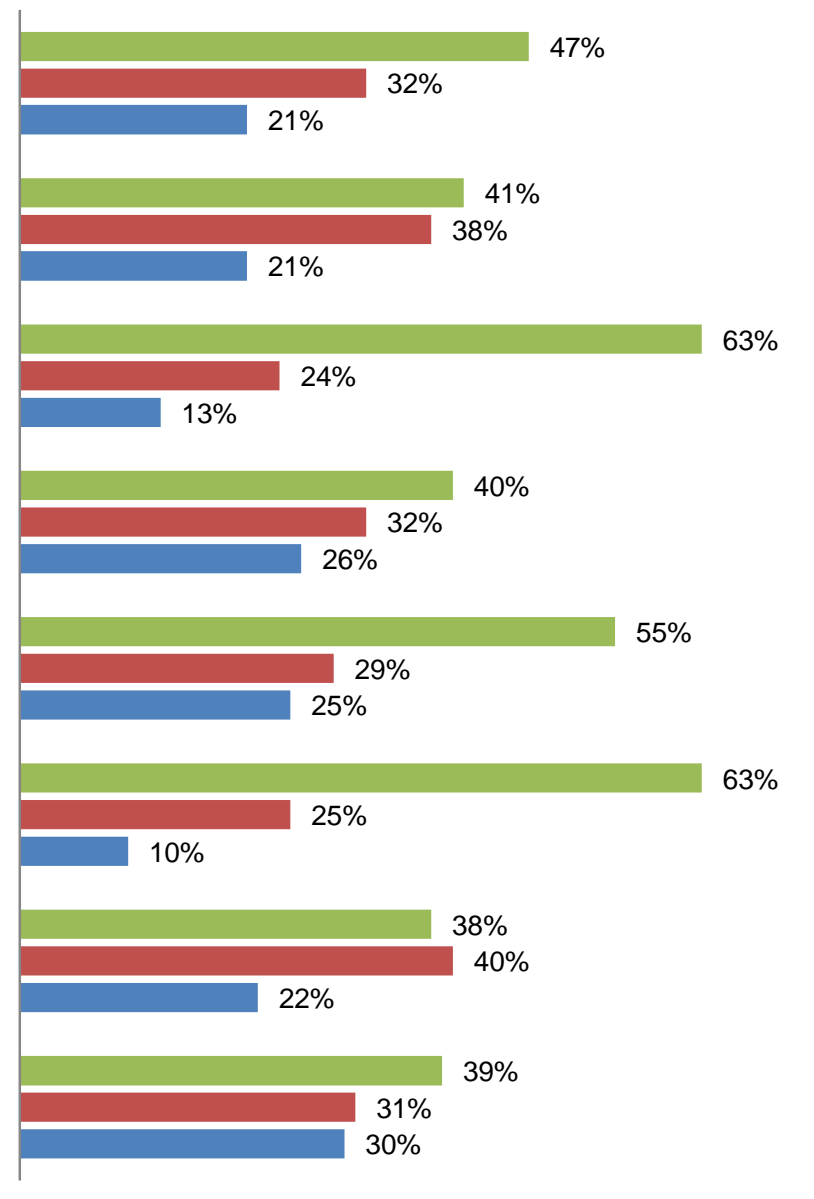

Fig. 1: Las mujeres estudiantes y su percepción de los medios de denuncia y campañas

El proceso de denuncia segura requiere que existan figuras de autoridad que inspiren confianza para contar situaciones relacionadas con el hostigamiento, para ellos se necesita que los directivos cuentan con los conocimiento necesarios para puedan brindar ayuda y puedan actuar de forma ética, profesional confidencial y oportuna, lo cual coincide en parte con Charmaraman, Jones, Stein y Espelage (2013).

Las campañas en contra de la violencia hacia las mujeres), se sugiere realizar programas con perspectivas de género acorde a las temporalidades por ejemplo talleres de verano, del día del amor y la amistad, platicas del día del padre, entre otros, lo cual coincide con Rubin y Borgers (1990) y Hill y Silva (2005). La Universidad (IPN- UPIICSA) en donde se realizó el estudio, cuenta con avances en materia del hostigamiento y acoso sexual, lo cual destaca que, a pesar de lo cuestionable de la efectividad en las campañas de difusión para prevenir, denunciar y sancionar el hostigamiento y acoso sexual, ya se tienen los cimientos de algo que puede llegar a ser la base para que las futuras generaciones de mujeres estudiantes conozcan la forma de cómo defenderse ante este tipo de actos hostiles y que ellas sean las multiplicadoras de la no violencia, del respeto y la tolerancia entre sus pares y no pares.

Las limitaciones que se tuvieron en este trabajo fueron diversas, la aplicación del instrumento no fue una tarea sencilla, a pesar de que la institución mantiene su campaña de prevención, existe muchos tabús alrededor del tema, por otro lado, se observó que los estudiantes varones estuvieron interesados en resolver el cuestionario, lo anterior se considerará para las siguientes investigaciones. Otro de los obstáculos es el escaso conocimiento del tema de parte de algunas autoridades y se percibe poco interés sobre las consecuencias del hostigamiento sexual en las alumnas. Por último, un obstáculo es encontrar entrevistas a profundidad de posibles víctimas de hostigamiento sexual para así obtener información que permita seguir con la investigación. 


\section{CONCLUSIONES}

Los resultados encontrados evidencian la invisibilidad de las políticas institucionales implementadas por el IPN relacionadas con el acoso sexual, los hallazgos permiten detectar que las acciones encaminadas a promover los mecanismos de la denuncia segura no están alcanzando con efectividad a las estudiantes y esto las ubica en zona de riego y por ende se incrementa su vulnerabilidad. El acoso sexual es una forma de violencia que se esconde, es silenciosa y lacerante, las víctimas viven en constante amenaza, para ayudarlas es necesario contar con grupos interdisciplinarios capaz de aplicar de forma rápida los protocolos de intervención, la impunidad y la falta de información relacionada con las sanciones son elementos que se combinan y pueden provocar que las mujeres no comiencen su proceso de denuncia.

Es imprescindible que cada unidad académica que conforma el Instituto Politécnico Nacional cuente con una oficina de gestión con perspectiva de género que sea capaz de atender y ofrecer apoyo para la difusión de una política para el manejo de acoso sexual, de promover tanto programas de formación como generar y vincular redes de apoyo, ya que se debe incluir también a los padres de familia. Dicha oficina deberá de contar con el apoyo del Departamento Jurídico de cada unidad académica. A su vez, se necesita fomentar la realización de investigaciones y estudios con perspectiva de género, en donde las autoridades puedan apoyar ampliamente, sin prejuicios, sin miedos, sin tabúes y sin máscaras.

La unidad académica analizada necesita, de forma urgente, retomar el programa de formación de tutores para que a través de ellos y de los padres de familia formen un frente común en contra del hostigamiento y acoso sexual. Es necesario también analizar los programas de estudio, implementar estrategias que favorezcan la transversalización de los temas de género por medio de las unidades de aprendizaje que así lo permitan. La aportación de este trabajo consiste en el reconocimiento del problema de la escasa evaluación y seguimiento que tiene los medios para denunciar el hostigamiento sexual, pues se observó que las políticas institucionales existentes no están llegando con una completa efectividad a las mujeres estudiantes del Instituto Politécnico Nacional.

Además es necesario que se adopten directamente en las unidades académicas, las estrategias establecidas en la Plataforma de Acción Beijing (1995:54) en donde se señala que deben de introducir sanciones penales, civiles, laborales y administrativas con el objetivo de castigar y reparar los daños causados a las mujeres víctimas de cualquier tipo de violencia. También enfatiza la importancia de insistir en la prevención de la violencia y el enjuiciamiento de los responsables, a su vez, el adoptar medidas para garantizar la protección de las mujeres víctimas de la violencia, por lo cual se debería de crear nuevas políticas en las escuelas para garantizar la protección de las estudiantes.

\section{REFERENCIAS}

Abuya, B. A., E. O. Onsomu, D. Moore y J. Sagwe, (2012). A phenomenological study of sexual harassment and violence among girls attending high schools in urban slums, Nairobi, Kenya. Journal of School Violence, 11(4), 323-344 (2012)

Angelone, D. J., D. Mitchell, y K. Carola. Tolerance of sexual harassment: A laboratory paradigm. Archives of sexual behavior, 38(6), 949-958 (2009)

Bond, M. A. Prevention and the ecology of sexual harassment: Creating empowering climates. Sexual assault and abuse: Sociocultural context of prevention, 147-173 (1995)

Bouquet, A., J.A. Cooper, A. Mingo y H. Moreno. Intrusas en la Universidad. México: Universidad Nacional Autónoma de México. Programa Universitario de Estudios de Género e Instituto de Investigaciones sobre la Universidad y la Educación (2013)

Cámara de Diputados del Honorable Congreso de la Unión. Ley General de Acceso de las Mujeres a una Vida Libre de Violencia, nueva Ley publicada en el Diario Oficial de la Federación el 1 de febrero de 2007 con su reforma publicada el 15 de enero de 2015 (2013)

Castañeda, M. y Castaaneda, M. El machismo invisible. México Ed. FDF: Grijalbo (2002)

Champion, D. R. (2006). Sexual Harassment: Criminal Justice and Academia.Criminal Justice Studies, 19(2), 101-109 (2002)

Charmaraman, L., A. E Jones, N. Stein y D.L. Espelage. Is it bullying or sexual harassment? Knowledge, attitudes, and professional development experiences of middle school staff. Journal of school health, 83(6), 438-444 (2013)

DeGue, S. Preventing Sexual Violence on College Campuses: Lessons from Research and Practice (2014) 
Dhlomo, T., R.M. Mugweni, G. Shoniwa, L. Maunganidze y T. Sodi (2012). Perceived Sexual Harassment Among Female Students at a Zimbabwean Institution of Higher Learning. Journal of Psychology in Africa, 22(2), 269-272 (2012)

Fasting, K., S. Chroni y N. Knorre. The experiences of sexual harassment in sport and education among European female sports science students. Sport, Education and Society, 19(2), 115-130 (2014)

Fitzgerald, L. F., M.J. Gelfand y F. Drasgow. Measuring sexual harassment: Theoretical and psychometric advances. Basic and Applied Social Psychology, 17(4), 425-445 (1995)

Fusilier, M, y C. Penrod. University Employee Sexual Harassment Policies. Employee Responsibilities and Rights Journal, 1-14 (2014)

Gelfand, M. J., L.F. Fitzgerald y F. Drasgow. The structure of sexual harassment: A confirmatory analysis across cultures and settings. Journal of Vocational Behavior, 47(2), 164-177 (1995)

Gross, E. y M. Mansour. ¿Qué es la teoría feminista?. Debate feminista, 85-105 (1995)

Hill, C. y H. Kearl. Crossing the Line: Sexual Harassment at School. American Association of University Women. 1111 Sixteenth Street NW, Washington, DC 20036 (2011)

Hill, C., y E. Silva. Drawing the Line: Sexual Harassment on Campus. American Association of University Women Educational Foundation, 1111 Sixteenth St. NW, Washington, DC 20036 (2005)

Jackman, M. R., Gender, violence, and harassment. In Handbook of the sociology of gender (275-317). Springer US. (2006)

Jones, T. S. y M.S. Remland. Sources of variability in perceptions of and responses to sexual harassment. Sex Roles, 27(3-4), 121-142 (1992)

Kalof, L., K.K Eby, J.L. Matheson y R.J Kroska. The influence of race and gender on student self-reports of sexual harassment by college professors. Gender \& Society, 15(2), 282-302 (2001)

Krauss, B. J., H.H. Krauss, J. O'Day y K. Rente. Sexual Violence in the Schools. In Violence in Schools (pp. 101-118). Springer US (2005)

Lonsway, K. A., L.M. Cortina y V.J Magley. Sexual harassment mythology: Definition, conceptualization, and measurement. Sex Roles, 58(9-10), 599-615 (2008)

Maguire, M. Sexual Harassment. Springer: Encyclopedia of Cross-Cultural School Psychology (2010)

Mitchell, K. J., M.L. Ybarra y J.D Korchmaros. Sexual harassment among adolescents of different sexual orientations and gender identities. Child abuse \& neglect, 38(2), 280-295 (2014)

Ochoa, L. M. Posibilidades y riesgos de las tutorías en la educación superior. Apuntes para el análisis. Quaderns digitals: Revista de nuevas tecnologías y sociedad, (51), 35 (2008)

Oliver, E. Women's choices shattered: Impact of gender violence on universities. Gendered Choices: Learning, Work, Identities in Lifelong Learning, 15, 69 (2011)

Osborne, B. Eliminating sexual violence in schools: implications for athletics administrators in the United States. The International Sports Law Journal, 13(1-2), 9-17 (2013)

Paludi, M., R. Nydegger, E. Desouza, L. Nydegger y K.A. Dicker. International perspectives on sexual harassment of college students. Annals of the New York Academy of Sciences, 1087(1), 103-120 (2006)

Pina, A., T.A. Gannon y B. Saunders. An overview of the literature on sexual harassment: Perpetrator, theory, and treatment issues. Aggression and Violent Behavior, 14(2), 126-138 (2009)

Presidencia de la República de los Estados Unidos Mexicanos. Programa Nacional para la Igualdad de Oportunidades y no Discriminación contra las mujeres 2013-2018. Diario Oficial de la Federación, Viernes 30 de agosto de 2013 (2014)

Presidencia de la República de los Estados Unidos Mexicanos. Plan Nacional de Desarrollo 2013-2018. Diario Oficial de la Federación, 20 de mayo de 2013 (2014)

Rahimi, R. y D. Liston. Race, class, and emerging sexuality: teacher perceptions and sexual harassment in schools. Gender and education, 23(7), 799-810 (2011)

Reilly, M. E., B. Lott y S.M. Gallogly. Sexual harassment of university students. Sex Roles, 15(7-8), 333-358 (1986)

Rodríguez, J. ¿Cómo fortalecer las instituciones de educación superior? en Gestión educativa y prospectiva humanística. México: Paidea, Siglo XXI (2013) 
Rosas, M. A. T. y S.O López. El Instituto Politécnico Nacional innovando en políticas en prevención de violencia con perspectiva de género. Innovación Educativa, 11(57), 195-205 (2011)

Rubin, L. J. y S.B. Borgers. Sexual harassment in universities during the 1980s. Sex Roles, 23(7), 397-411 (1990)

Sbraga, T. P. y W. O'donohue. Sexual harassment. Annual review of sex research, 11(1), 258-285 (2000)

Simon, B. L. The feminization of poverty: A call for primary prevention, Journal of Primary Prevention, 9(1-2), 6-17 (1988)

Tang, C. S. K., M.S. Yik, F.M. Cheung, P.K. Choi y K.C. Au. Sexual harassment of Chinese college students. Archives of sexual behavior, 25(2), 201-215 (1996)

Thomas, A. M. Politics, policies and practice: assessing the impact of sexual harassment policies in UK universities. British journal of sociology of education, 25(2), 143-160 (2004)

Vázquez, F. L., A. Torres y P. Otero. Gender-based violence and mental disorders in female college students. Social psychiatry and psychiatric epidemiology, 47(10), 1657-1667 (2012)

Yin, R. K. Case study research: Design and methods. Sage publications (2013) 
